\title{
The almost fixed point property is not invariant under isometric renormings
}

\section{J. Villada Bedoya ${ }^{1}$ (D)}

Received: 28 May 2020 / Accepted: 1 March 2021 / Published online: 20 March 2021

(c) The Author(s) 2021

\section{Abstract}

In the present note we prove the non set-stability of the AFPP under isometric renormings in the setting of Banach spaces containing a complemented isomorphic copy of $c_{0}$ or $\ell_{1}$

Keywords Banach-Mazur distance $\cdot$ Almost fixed point property $\cdot$ Set-stability · Complemented isomorphic copy of $c_{0}$ and $\ell_{1} \cdot$ Non-reflexive space $\cdot$ Isometric renormings

Mathematics Subject Classification 47H09 - 46B20

\section{Introduction}

Let $X$ be a Banach space which has a property $\mathcal{P}$. One of the fundamental problems in metric fixed point theory is to determine if $\mathcal{P}$ is stable, that is, if the property $\mathcal{P}$ is shared by all the spaces 'near enough' to $X$. To make precise the problem of stability usually we consider the following definition of distance introduced by Stefan Banach in [1] and known as Banach-Mazur distance.

Definition 1 Let $X$ and $Y$ be Banach spaces. The Banach-Mazur distance between $X$ and $Y$, denoted by $d(X, Y)$ is defined as:

$$
d(X, Y)=\inf \left\{\|T\| \cdot\left\|T^{-1}\right\|: T \text { is an isomorphism from } X \text { to } Y\right\} .
$$

When $X$ and $Y$ are not isomorphic, we say that $d(X, Y)=\infty$.

We say that $\mathcal{P}$ is stable if for every Banach space $X$ which satisfies $\mathcal{P}$, there exists $\gamma>0$ such that $d(X, Y)<\gamma$ implies that $Y$ verifies $\mathcal{P}$. All those properties that are invariant under isomorphism are stable in this sense, for instance reflexivity, super reflexivity, BanachSaks property, Krein-Milman property, Radon-Nykodým property, Shur property, property of being a weakly compactly generated space, stable weak* fixed point property $([2,3])$, uniform normal structure, uniform non-squareness among others.

\section{J. Villada Bedoya}

jeimer.villada@cimat.mx

1 Institute of Mathematics, Marie Curie Sklodowska University, Pl. Marii Curie-Sklodowskiej 1, 20-031 Lublin, Poland 
Since by definition $d(X, Y) \geq 1$ we can restrict the mentioned notion of stability to the case in which $d(X, Y)=1$.

Definition 2 We say that a property $\mathcal{P}$ is invariant under Banach-Mazur distance 1 if for every pair of Banach spaces $X, Y$, with $d(X, Y)=1, X$ has the property $\mathcal{P}$ if and only if $Y$ has the property $\mathcal{P}$.

In [17] $Ł$. Piasecki proved that the weak* fixed point property $\left(w^{*}\right.$-fpp), $w^{*}$-normal structure $\left(w^{*}-\mathrm{NS}\right)$, the weak* Kadec-Klee property $\left(w^{*}-\mathrm{KK}\right)$, the weak* Generalized GossezLami Dozo property ( $w^{*}$-GGLD), the finite dimensional norm preserving extension property (FNEP) and the compact norm preserving extension property (CNEP) are not invariant under Banach-Mazur distance 1, even in the framework of separable Lindenstrauss spaces. In the general setting of Banach spaces, he proved also that uniform convexity in every direction, local uniform rotundity, uniform smoothness, the Kadec-Klee property, the weak*-Opial property, the Opial property, the weak fixed point property and weak normal structure are not invariant under Banach-Mazur distance 1.

Let $C$ be a closed convex subset of a Banach space $X$ and $T: C \rightarrow C$ a nonexpansive function, that is, for every $x, y \in C,\|T x-T y\| \leq\|x-y\|$. Whenever inf $\{\|T x-x\|: x \in$ $C\}=0$, we say that $C$ satisfies the almost fixed point property (AFPP). In relation to this concept, in [9] it was studied the following notion of stability:

Definition 3 We denote by $\mathcal{C}(X,\|\cdot\|)$ the collection of closed convex sets with the AFPP in $(X,\|\cdot\|)$. We will say that $(X,\|\cdot\|)$ has stability of the AFPP if for any norm $\|\cdot\|_{1}$ on $X$ equivalent to $\|\cdot\|$, we have that $\mathcal{C}(X,\|\cdot\|)=\mathcal{C}\left(X,\|\cdot\|_{1}\right)$. If $\|\cdot\|_{1}$ is a norm on $X$ equivalent to $\|\cdot\|$, we say that the collections of sets with the AFPP in $(X,\|\cdot\|)$ and $\left(X,\|\cdot\|_{1}\right)$ differ when $\mathcal{C}(X,\|\cdot\|) \neq \mathcal{C}\left(X,\|\cdot\|_{1}\right)$.

Goebel and Kuczumow in [13] proposed the problem of characterizing those closed convex subsets of a Banach space verifying the AFPP. Regarding this, a result obtained by S. Reich in [18] establishes that if $X$ is a reflexive space, a closed convex set $C \subset X$ has the AFPP if and only if $C$ is linearly bounded (it contains no ray). Observe that the condition of linearly boundedness is invariant under isomorphisms and consequently if $(X,\|\cdot\|)$ is a reflexive space, $X$ has stability of the AFPP.

Afterwards in [19] I. Shafrir introduced the concept of directionally boundedness and proved, without assumptions of reflexivity, that a closed convex subset $C$ of a Banach space $X$ has the AFPP if and only if $C$ is directionally bounded. In the last years techniques involving the AFPP have been very useful in the study of various problems in fixed point theory $[4,7,8,10,15]$.

Recently in [9] the authors proved the following characterization of reflexivity in terms of the AFPP:

Theorem 1 Let $X$ be a Banach space. Then $X$ is reflexive if and only if $X$ has stability of the AFPP. Moreover, if $(X,\|\cdot\|)$ is not reflexive, for every $\delta>0$, there exists an equivalent norm $|\cdot|$ in $X$ such that $d((X,\|\cdot\|),(X,|\cdot|))<1+\delta$ and the respective collections of sets with the AFPP differ.

Considering the problem posed by Piasecki in [17] of determining geometrical properties that are not invariant under Banach-Mazur distance 1, we propose the following strengthening of definition 1.1 in [9]:

Definition 4 We say that a Banach space $(X,\|\cdot\|)$ has set-stability of the AFPP under isometric renormings, if for every norm $|\cdot|$ on $X$ such that $(X,\|\cdot\|)$ and $(X,|\cdot|)$ are isometric spaces, we have that $\mathcal{C}(X,\|\cdot\|)=\mathcal{C}(X,|\cdot|)$. 
Observe that every reflexive Banach space has set-stability of the AFPP under isometric renormings.

Bearing in mind Definition 4 and the last conclusion in Theorem 1, we can ask if any Banach space has set-stability of the AFPP under isometric renormings.

The purpose of the present note is to give a negative answer to this question by showing a wide class of spaces which have not set-stability of the AFPP under isometric renormings.

\section{Preliminaries}

In [19] I. Shafrir introduced the following concept:

Definition 5 A sequence $\left(x_{n}\right)$ in a Banach space $X$ is called a directional sequence if:

(i) $\left\|x_{n}\right\| \rightarrow \infty$

(ii) There is $b \geq 0$ such that for all $n_{1}<n_{2}<\cdots<n_{l}$

$$
\left\|x_{n_{1}}-x_{n_{l}}\right\| \geq \sum_{i=1}^{l-1}\left\|x_{n_{i}}-x_{n_{i+1}}\right\|-b .
$$

He called a closed convex set $C \subset X$ directionally bounded if it contains no directional sequences and proved the following criterium to determine when $C$ verifies this property.

Theorem 2 A closed convex set $C$ in a Banach space $X$ is directionally bounded if for every sequence $\left\{x_{n}\right\}$ in $C$ such that $\left\|x_{n}\right\| \rightarrow \infty$ and for every $f$ in the unitary ball of $X^{*}$,

$$
\limsup _{n} f\left(x_{n} /\left\|x_{n}\right\|\right)<1 .
$$

In the same work he showed that the condition of directionally boundedness is equivalent to the AFPP in Banach spaces.

Theorem 3 A closed convex set $C$ in a Banach space $X$ has the AFPP if and only if it is directionally bounded.

Using some of the tools developed by Shafrir in his study of the AFPP, in [9] the authors proved the following characterization for reflexive spaces in terms of the AFPP.

Theorem 4 Let $(X,\|\cdot\|)$ be a non-reflexive Banach space. Then for every $\epsilon>0$ there is a renorming $(X,|\cdot|)$ with $d((X,\|\cdot\|),(X,|\cdot|))<1+\epsilon$ such that the respective families of closed convex subsets with the AFPP differ.

The space of sequences of summable modulus and the space of sequences converging to 0 are denoted respectively by $\ell_{1}$ and $c_{0}$. If we write $\ell_{1}$ or $c_{0}$ it is understood that $\ell_{1}=\left(\ell_{1},\|\cdot\|_{1}\right)$ and $c_{0}=\left(c_{0},\|\cdot\|_{\infty}\right)$ where $\|x\|_{1}=\sum_{i=1}^{\infty}|x(i)|$ and $\|x\|_{\infty}=\sup _{i \in \mathbb{N}}|x(i)|$.

The following results give conditions under which a (isomorphic) copy of $c_{0}$ or $\ell_{1}$ is complemented in a Banach space $X$. We include them in order to exemplify some applications of our main result. The first result is an immediate consequence of Proposition 1.8 in [14].

Theorem 5 Let $X$ be a Banach space $X$ with an unconditional basis. Then every copy of $\ell_{1}$ in $X$ contains a complemented copy of $\ell_{1}$ (complemented in $X$ ). 
Theorem 6 ([5, Theorem 2.2]) Let $X$ be a real Banach space that does not contain a copy of $\ell_{1}$. If $X$ contains a copy of $c_{0}$, then $X$ contains a complemented isomorphic copy of $c_{0}$.

Theorem 7 ([6, Theorem 10 p. 48]) Let X be a Banach space. Then $X^{*}$ contains an isomorphic copy of $c_{0}$ if and only if $X$ contains a complemented isomorphic copy of $\ell_{1}$.

Theorem 8 ([12, Corollary 2.2]) Let $X$ be a separable Banach space and let $Y$ be a subspace of $X$ which is isomorphic to $c_{0}$. Then $Y$ is complemented in $X$.

Throughout this work we consider only real Banach spaces. We denote by $\mathcal{P}(X)$ the collection of equivalent norms to a fixed norm of a Banach space $X$.

\section{Non stability of the AFPP under Banach-Mazur distance 1}

We start proving our main result for the particular cases of $\ell_{1}$ and $c_{0}$.

Lemma $1 \operatorname{Let}(X,\|\cdot\|)=\ell_{1}$ or $(X,\|\cdot\|)=c_{0}$. Then there is a norm $\|\cdot\|_{1} \in \mathcal{P}(X)$ such that $\left(X,\|\cdot\|_{1}\right)$ has not set-stability of the AFPP under isometric renormings.

Proof Let $\left(e_{n}\right)$ denote the canonical basis of $X$ and let $W=\left[e_{2 n-1}\right]$, where $\left[e_{2 n-1}\right]$ denotes the closed linear span of $\left\{e_{2 n-1}: n \in \mathbb{N}\right\}$. By Theorem 4 there is an equivalent norm $|\|\cdot\|| \in \mathcal{P}(W)$ such that the collections of directionally bounded sets in $(W,\|\cdot\|)$ and $(W,|\|\cdot\||)$ differ, so without loss of generality we may assume that there is a closed convex unbounded set $C \subset W$ which is directionally bounded with respect to the norm $\|\cdot\|$ but it is not with respect to the norm $|\|\cdot\||$. Let $P: X \rightarrow W$ be such that if $x=\sum_{n=1}^{\infty} a_{n} e_{n} \in X$, then $P x=\sum_{n=1}^{\infty} a_{2 n-1} e_{2 n-1}$. Define $\|\cdot\|_{1}$ and $\|\cdot\|_{2} \in \mathcal{P}(X)$ such that

$$
\|x\|_{1}=\|P x\|+|\|(I-P) x\||
$$

and

$$
\|x\|_{2}=|\|P x\||+\|(I-P) x\| .
$$

Since the inclusions $i_{1}:(W,\|\cdot\|) \rightarrow\left(X,\|\cdot\|_{1}\right)$ and $i_{2}:(W,|\|\cdot\||) \rightarrow\left(X,\|\cdot\|_{2}\right)$ are isometries on their images, $C$ has the AFPP with respect to the norm $\|\cdot\|_{1}$, but it has not the AFPP for the norm $\|\cdot\|_{2}$. Observe that if $x=\sum_{n=1}^{\infty} a_{n} e_{n} \in X$, the function $T:\left(X,\|\cdot\|_{1}\right) \rightarrow\left(X,\|\cdot\|_{2}\right)$ defined as

$$
(T x)(i)= \begin{cases}a_{2 j-1}, & \text { if } i=2 j \text { for some } j \in \mathbb{N}, \\ a_{2 j}, & \text { if } i=2 j-1 \text { for some } j \in \mathbb{N},\end{cases}
$$

is an onto linear isometry.

We can generalize the conclusion in Lemma 1 to the class of spaces containing a complemented subspace isomorphic to $c_{0}$ or $\ell_{1}$.

Theorem 9 Let $(X,\|\cdot\|)$ be a Banach space containing a complemented isomorphic copy of $c_{0}$ or $\ell_{1}$. Then there is $\|\cdot\|_{1} \in \mathcal{P}(X)$ such that $\left(X,\|\cdot\|_{1}\right)$ has not set-stability of the AFPP under isometric renormings.

Proof Let $V$ be a complemented subspace of $X$ isomorphic to $Y$, where $Y$ is $c_{0}$ or $\ell_{1}$ and denote by $|\cdot|_{1},|\cdot|_{2}$ the two norms on $Y$, garanteed by Lemma 1 , such that the families of sets 
with the AFPP in $\left(Y,\left.|\cdot|\right|_{1}\right)$ and $\left(Y,|\cdot|_{2}\right)$ differ. Let $T_{1}: V \rightarrow\left(Y,|\cdot|_{1}\right)$ and $T_{2}: V \rightarrow\left(Y,|\cdot|_{2}\right)$ be isomorphisms. If we consider on $V$ the norms $|\|\cdot\||_{1}$ and $|\|\cdot\||_{2} \in \mathcal{P}(V)$ such that

$$
\|\| x \|\left.\right|_{1}=\left|T_{1} x\right|_{1} \text { and }\left.\quad\|x\|\right|_{2}=\left|T_{2} x\right|_{2},
$$

then $R_{1}:\left(V,|\|\cdot\||_{1}\right) \rightarrow\left(Y,|\cdot|_{1}\right)$ and $R_{2}:\left(V,|\|\cdot\||_{2}\right) \rightarrow\left(Y,|\cdot|_{2}\right)$ such that $R_{i} x=T_{i} x$, $i=1,2$, are linear onto isometries. Let $T:\left(Y,|\cdot|_{1}\right) \rightarrow\left(Y,|\cdot|_{2}\right)$ be the isometry considered in Lemma 1 . Since $T$ is an onto linear isometry, we have that $L:\left(V,|\|\cdot\||_{1}\right) \rightarrow\left(V,|\|\cdot\||_{2}\right)$ defined as $L=R_{2}^{-1} T R_{1}$ is an onto linear isometry. Let $P: X \rightarrow V$ be a linear bounded projection from $X$ onto $V$ and define $\|\cdot\|_{1},\|\cdot\|_{2} \in \mathcal{P}(X)$ such that

$$
\|x\|_{1}=|\|P x\||_{1}+\|(I-P) x\|
$$

and

$$
\|x\|_{2}=\|\| P x\left\|_{2}+\right\|(I-P) x \| .
$$

If $S:\left(X,\|\cdot\|_{1}\right) \rightarrow\left(X,\|\cdot\|_{2}\right)$ is defined as $S x=(I-P) x+L P x$ it is easy to check that $S$ is an isomorphism and furthermore

$$
\begin{aligned}
\|S x\|_{2} & =\|\| P L P x\|\mid+\|(I-P) x\|=\|\|L P x\|_{2}+\|(I-P) x\| \\
& =\|P x\|_{1}+\|(I-P) x\|=\|x\|_{1},
\end{aligned}
$$

since $L$ is an isometry. Cleary $\mathcal{C}\left(X,\|\cdot\|_{1}\right) \neq \mathcal{C}\left(X,\|\cdot\|_{2}\right)$.

Below we show a wide variety of cases in which we can apply Theorem 9.

Lemma 2 Let $X$ be a non reflexive Banach space that satisfies any of the following conditions:

(i) $X$ has an unconditional Schauder Basis.

(ii) $X$ contains an isomorphic copy of $c_{0}$ and $X$ does not contain an isomorphic copy of $\ell_{1}$.

(iii) $X$ is separable and contains an isomorphic copy of $c_{0}$.

(iv) $X^{*}$ contains an isomorphic copy of $c_{0}$.

(v) $X$ has an uncountable unconditional Schauder basis [11] and contains an isomorphic copy of $\ell_{1}$.

Then there is a norm $\|\cdot\|_{1} \in \mathcal{P}(X)$ such that $\left(X,\|\cdot\|_{1}\right)$ has not set-stability of the AFPP under isometric renormings.

Proof (i) By James theorem ([16, Corollary 4.4.23]) $X$ contains a subspace $Y$ isomorphic to $c_{0}$ or $\ell_{1}$. If $Y$ is isomorphic to $c_{0}$, Theorem 8 implies that $Y$ is complemented. If $Y$ is isomorphic to $\ell_{1}$, by Theorem 5 we can assume that $Y$ is complemented. So in either case, the hypotheses of Theorem 9 are satisfied. Similarly we prove (ii)-(v) using Theorem 6, Theorem 8, Theorem 7 and Theorem 1a in [11] respectively.

Lemma 2 offers a large class of examples of Banach spaces in which we can conclude the non set-stability of the AFPP under isometric renormings. However, it is still possible that given a Banach space $X$, we can find two non-isometric norms $\|\cdot\|_{1},\|\cdot\|_{2}$ in $\mathcal{P}(X)$, such that $\left(X,\|\cdot\|_{1}\right)$ and $\left(X,\|\cdot\|_{2}\right)$ share the collection of sets with the AFPP and $d\left(\left(X,\|\cdot\|_{1}\right),(X, \| \cdot\right.$ $\left.\left.\|_{2}\right)\right)=1$. In the following, we illustrate this situation in the particular case of $X=c_{0}$.

Lemma 3 Consider the spaces $X=\left(c_{0},\|\cdot\|\right)$ and $Y=\left(c_{0},|\|\cdot\||\right)$ where

$$
\|x\|=\|x\|_{\infty}+\sum_{j=1}^{\infty} \frac{|x(j)|}{2^{j}}
$$


and

$$
|\|x\||=\|x\|_{\infty}+\sum_{j=2}^{\infty} \frac{|x(j)|}{2^{j-1}}
$$

then $d(X, Y)=1$.

Proof Let $T_{n}: X \rightarrow Y$ be such that

$$
T_{n}(x)=(x(n), x(1), \ldots, x(n-1), x(n+1), \ldots) .
$$

It is easy to see that $T_{n}$ is an isomorphism such that $\left\|T_{n}\right\| \rightarrow 1$ and $\left\|T_{n}^{-1}\right\| \rightarrow 1$.

We want to compare the collections of directionally bounded sets of the spaces $X$ and $Y$ defined in Lemma 3. In order to do this, by Theorem 2, it is very useful to know $X^{*}$ and $Y^{*}$. The space $X^{*}$ was described in [4], so we proceed to determine $Y^{*}$. Straightforward calculations allow us to prove the following result:

Proposition 1 Let $N \in \mathbb{N}, Z_{N}=\{x \in Y: x(i)=0, \forall i>N\}$ and $Y_{N}=\left(Z_{N},|\|\cdot\||_{\left.\right|_{Z_{N}}}\right)$. Then the set of extreme points with non-negative coordinates in the unitary ball of $Y_{N}$ (denoted as $\xi^{+}\left(Y_{N}\right)$ ) satisfies:

$$
\xi^{+}\left(Y_{N}\right) \subset\left\{\left(\frac{1}{\sum_{i \in F} \frac{1}{2^{i-1}}}\right) \sum_{i \in F} e_{i}: F \subset\{1, \ldots, N\}, 1 \in F\right\} .
$$

Where $\left(e_{n}\right)$ is the canonical basis of $c_{0}$.

From the last proposition it follows that:

Proposition $2\left(c_{0},|\|\cdot\||\right)^{*}=\left(\ell_{1},\|\cdot\|_{*}\right)$ where for $f=\left(c_{i}\right) \in \ell_{1}$

$$
\|f\|_{*}=\sup _{\substack{F \subset \mathbb{N} \\ 1 \in F, \# F<\infty}}\left(\frac{1}{\sum_{i \in F} \frac{1}{2^{i-1}}}\right) \sum_{i \in F}\left|c_{i}\right| .
$$

The next proposition gives a criterium to distinguish between directional and non directional sequences in the space $\left(c_{0},|\|\cdot\||\right)$. Bearing in mind Proposition 2, the proof of this result is analogous to the proof of proposition 9 in [4].

Proposition 3 Let $C$ be a closed convex unbounded set in $c_{0}$. Suppose that $C$ is directionally bounded in $\left(c_{0},|\|\cdot\||\right)$. Let $\left(x_{n}\right) \subset C$ be a sequence such that $\lim _{n \rightarrow \infty}\left\|x_{n}\right\|_{\infty}=\infty$, with $x_{n}=\left(x_{n}(k)\right)_{k=1}^{\infty}$. Then for every $n_{0}, k_{0} \in \mathbb{N}$ there exist $n>n_{0}$ and $k>k_{0}$ such that $\left\|x_{n}\right\|_{\infty}=\left|x_{n}(k)\right|$.

The following result describes a sufficient condition in $c_{0}$ for an unbounded sequence not to be directional.

Proposition 4 (Proposition 10 in [4]) Let $\left(x_{n}\right) \subset c_{0}$ be a sequence such that $\lim _{n \rightarrow \infty}\left\|x_{n}\right\|_{\infty}=$ $\infty$. If for every $n_{0}, k_{0} \in \mathbb{N}$ there exists $n>n_{0}$ and $k>k_{0}$ such that $\left\|x_{n}\right\|_{\infty}=\left|x_{n}(k)\right|$, then $\left(x_{n}\right)$ is not a directional sequence in $\left(c_{0},\|\cdot\|_{\infty}\right)$.

Using Propositions 3 and 4 we can establish a relationship between the collections of directionally bounded sets in $\left(c_{0},|\|\cdot\||\right)$ and $c_{0}$. 
Theorem 10 Let $C$ be a convex, closed and unbounded subset of $c_{0}$. Then $C$ is directionally bounded in $\left(c_{0},\|\cdot\|_{\infty}\right)$ if and only if it is directionally bounded in $\left(c_{0},|\|\cdot\||\right)$.

Proof Let $C \subset\left(c_{0},|\|\cdot\||\right)$ be a closed convex unbounded and directionally bounded set and $\left(x_{n}\right) \subset C$ a sequence such that $\left|\left\|x_{n}\right\|\right| \rightarrow \infty$. By Proposition 3 for every $n_{0}, k_{0} \in \mathbb{N}$ there are $n>n_{0}$ and $k>k_{0}$ such that $\left\|x_{n}\right\|_{\infty}=\left|x_{n}(k)\right|$ and Proposition 4 implies that $\left(x_{n}\right)$ is not a directional sequence in $\left(c_{0},\|\cdot\|_{\infty}\right)$.

Conversely, suppose that $C \subset\left(c_{0},\|\cdot\|_{\infty}\right)$ is a closed convex and unbounded set. If $\left(x_{n}\right)$ is a directional sequence in $\left(c_{0},|\|\cdot\||\right)$. Take $b \geq 0$ as given by the definition of directional sequence. Let $n_{1}<n_{2}<\cdots<n_{s}$. Then

$$
\begin{aligned}
b \geq & \sum_{i=1}^{s-1}\left|\left\|x_{n_{i}}-x_{n_{i+1}}\right\|\right|-\left|\left\|x_{n_{s}}-x_{n_{1}}\right\|\right| \\
= & \sum_{i=1}^{s-1}\left\|x_{n_{i}}-x_{n_{i+1}}\right\|_{\infty}-\left\|x_{n_{s}}-x_{n_{1}}\right\|_{\infty} \\
& +\sum_{i=1}^{s-1}\left(\sum_{m=2}^{\infty} \frac{\left|x_{n_{i+1}}(m)-x_{n_{i}}(m)\right|}{2^{m-1}}\right)-\sum_{m=2}^{\infty} \frac{\left|x_{n_{1}}(m)-x_{n_{s}}(m)\right|}{2^{m-1}} .
\end{aligned}
$$

By the triangle inequality:

$$
\sum_{m=2}^{\infty} \frac{1}{2^{m-1}}\left(\sum_{i=1}^{s-1}\left|x_{n_{i+1}}(m)-x_{n_{i}}(m)\right|-\left|x_{n_{1}}(m)-x_{n_{s}}(m)\right|\right) \geq 0 .
$$

From this:

$$
\sum_{i=1}^{s-1}\left\|x_{n_{i+1}}-x_{n_{i}}\right\|_{\infty}-\left\|x_{n_{1}}-x_{n_{s}}\right\|_{\infty} \leq b
$$

and $\left(x_{n}\right)$ is a directional sequence in $\left(c_{0},\|\cdot\|_{\infty}\right)$, so if $C$ is directionally bounded in $\left(c_{0},\|\cdot\|_{\infty}\right)$, it is in $\left(c_{0},|\|\cdot\||\right)$.

Finally, from the above theorem, we conclude that there exist two equivalent renormings of a Banach space $X$ whose Banach-Mazur distance is 1 and they share the collection of sets with the AFPP.

Corollary 1 A closed convex set $C \subset c_{0}$ has the AFPP in $\left(c_{0},\|\cdot\|\right)$ if and only if it has the AFPP in $\left(c_{0},|\|\cdot\||\right)$. Moreover, $d\left(\left(c_{0},\|\cdot\|\right),\left(c_{0},|\|\cdot\||\right)\right)=1$.

Proof Theorem 11 in [4] implies that $C$ is directionally bounded in $\left(c_{0},\|\cdot\|\right)$ if and only if $C$ is directionally bounded in $c_{0}$. The conclusion follows from Theorems 3 and 10 . The last statement was proved in Lemma 3.

Acknowledgements The research of the author has been partially supported by SEP-CONACYT Grant A1S-53349The author would like to thank professor T. Domínguez Benavides for his interesting questions on the subject and professor H. Fetter Nathansky for her valuable comments and suggestions during the process of writing and reviewing this note. The author would also like to thank the referees for their helpful comments that led to the improved version of the manuscript. 
Open Access This article is licensed under a Creative Commons Attribution 4.0 International License, which permits use, sharing, adaptation, distribution and reproduction in any medium or format, as long as you give appropriate credit to the original author(s) and the source, provide a link to the Creative Commons licence, and indicate if changes were made. The images or other third party material in this article are included in the article's Creative Commons licence, unless indicated otherwise in a credit line to the material. If material is not included in the article's Creative Commons licence and your intended use is not permitted by statutory regulation or exceeds the permitted use, you will need to obtain permission directly from the copyright holder. To view a copy of this licence, visit http://creativecommons.org/licenses/by/4.0/.

\section{References}

1. Banach, S.: Théorie des opérations linéaires. Warszawa (1932)

2. Casini, E., Miglierina, E., Piasecki, L., Popescu, R.: Stability constants of the weak* fixed point property in the space $\ell_{1}$. J. Math. Anal. Appl. 452(1), 673-684 (2017)

3. Casini, E., Miglierina, E., Piasecki, L., Popescu, R.: Weak* fixed point property in $\ell_{1}$ and polihedrality in Lindenstrauss spaces. Studia Math. 241, 159-172 (2018)

4. Castillo, E., Fetter, H., Gamboa, B., Núñez, F.: Directionally bounded sets in $c_{0}$ with equivalent norms. J. Math. Anal. Appl. 419(2), 727-737 (2014)

5. Díaz, S., Fernández, A.: Reflexivity in Banach lattices. Arch. der Mathematik. 63(6), 549-552 (1994)

6. Diestel, J.: Sequences and Series in Banach Spaces. Science \& Business Media, Berlin (2012)

7. Domínguez, T.: On the failure of the fixed point property for some unbounded subsets of $\ell_{1}$. J. Nonlinear Convex Anal. 17(7), 1249-1257 (2016)

8. Domínguez, T.: The failure of the fixed point property for unbounded sets in $c_{0}$. Proc. Am. Math. Soc. 140(2), 645-650 (2012)

9. Fetter, H., Japón, M., Villada Bedoya, J.: Reflexivity is equivalent to stability of the almost fixed point property. J. Math. Anal. Appl. 459(2), 789-796 (2018)

10. Fetter, H., Villada Bedoya, J.: Some considerations about the fpp and the AFPP in $c_{0}$ with an equivalent norm. J. Nonlinear Convex Anal. 17(11), 2317-2327 (2016)

11. Finol, C., Wójtowicz, M.: Complemented copies of $\ell_{1}$ in Banach spaces with an unconditional basis. J. Math. Anal. Appl. 342, 83-88 (2008)

12. Gilles, G.: The Banach space $c_{0}$. Extracta Math. 16, 1-25 (2001)

13. Goebel, K., Kuczumow, T.: A contribution to the theory of nonexpansive mappings. Calcutta Math. Soc. 70(1), 978 (1978)

14. Linberg, K.: On subspaces of Orlicz sequences spaces. Stud. Math. 45, 119-146 (1973)

15. Matouskova, E., Reich, S.: Reflexivity and approximate fixed pointsS. Stud. Math. 159, 403-415 (2003)

16. Megginson, R.: An Introduction to Banach Space Theory. Springer Science \& Business Media, Berlin (2012)

17. Piasecki, L.: On Banach space properties that are not invariant under Banach-Mazur distance 1. J. Math. Anal. Appl. (2018). https://doi.org/10.1016/j.jmaa.2018.07.063

18. Reich, S.: The almost fixed point property for nonexpansive mappings. Proc. Am. Math. Soc. 88(1), 44-46 (1983)

19. Shafrir, I.: The approximate fixed point property in Banach and hyperbolic spaces. Israel J. Math. 71(2), 211-223 (1990)

Publisher's Note Springer Nature remains neutral with regard to jurisdictional claims in published maps and institutional affiliations. 